\title{
Depresión, ansiedad y suicidalidad en una población de Lima Metropolitana durante la pandemia por COVID-19
}

\section{Depression, anxiety and suicidality in a population of Metropolitan Lima during the COVID-19 pandemic}

\author{
Sergio R. Seminario-Huallpa' \\ Consuelo Luna-Muñoz ${ }^{2}$ \\ Jhony A. De La Cruz-Vargas ${ }^{3}$
}

\begin{abstract}
Seminario-Huallpa S.R, Luna-Muñoz C, Jhony A. De La Cruz-Vargas JA. Depresión, ansiedad y suicidalidad en una población de Lima Metropolitana durante la pandemia por COVID-19. Rev Soc Peru Med Interna. 2021;34(4): I46-I53. DOI: https://doi.org/10.36393/ spmi.v34i4.630
\end{abstract}

\begin{abstract}
RESUMEN
Objetivos: Determinar la frecuencia y los factores asociados a depresión, ansiedad y suicidalidad en una población de Lima Metropolitana durante la pandemia por el virus SARS-CoV2. Material y métodos: Estudio observacional, transversal y analítico; el tipo de muestreo fue no probabilístico en "bola de nieve", se utilizaron dos encuestas virtuales, la Escala de Psicopatología Depresiva-6 y la Escala de Ansiedad de Lima-20. Se calcularon razones de prevalencias crudas (RP) y ajustadas (RP) con intervalos de confianza al $95 \%$ (IC $95 \%$ ) por regresiones de Poisson con varianza robusta. Las variables con valores de $\mathrm{p}<0,10$ se incluyeron en el análisis multivariado. Resultados: El $12,4 \%$ presentó depresión, 23, I\% ansiedad y 5,3\% suicidalidad. En el modelo multivariado se encontró asociación significativa entre ser divorciado(a) / viudo (a) / separado(a) (RP =2, I9; IC: I, I 8-4,07), tener estudios superiores completos $(R P=0,44 ; I C: 0,28-0,69)$ e incompletos $(R P=0,26 ; I C: 0,14-0,48)$ y no tener trabajo $(R P=3,5 I ; I C: 0,79-I 0,22)$ con depresión. Hubo asociación significativa entre género femenino $(R P=I, 62 ; I C: I, 25-2,09)$, ser joven $(R P=3,24$; IC: $I, 34-7,83)$, ser adulto $\left(R P_{a}=3,48 ; I C: I, 43-8,46\right)$, tener estudios superiores completos $\left(R P_{a}=0,44 ;{ }^{a} I C: 0,33-0,58\right)$ y ser trabajador independiente $(R P=2,94 ; I C: I, 37-6,29)$ con ansiedad. El género femenino (RP =0,48; IC:0,27-0,85), ser divorciado(a)/viudo(a)/separado(a) $(R P=4,69 ; I C: 2,23-9,83)$ y ser trabajador dependiente $(R P=0,24 ; I C: 0,10-0,57)$ fueron factores asociados a suicidalidad. Conclusiones: El tipo de ocupación estuvo asociado significativamente a depresión, ansiedad y suicidalidad; el género, a ansiedad y suicidalidad; el estado civil, a depresión y suicidalidad; el grado de instrucción, a depresión y ansiedad; y, el rango etario, a ansiedad.
\end{abstract}

Palabras claves: depresión, ansiedad, ideación suicida, aislamiento social, virus SARS-CoV-2.

\section{ABSTRACT}

Objectives:To determine the frequency and factors associated with depression, anxiety and suicidality in a population of Lima Metropolitan Area during the SARSCoV-2 pandemic. Materials and methods: Observational, cross-sectional and analytical study; the type of sampling was non-probabilistic "snowball", two virtual surveys were used, the Depressive Psychopathology Scale-6 and the Lima Anxiety Scale-20. Crude (PRc) and adjusted (PRa) prevalence ratios with 95\% confidence intervals $(95 \% \mathrm{Cl})$ were calculated by Poisson regressions with robust variance. Variables with p-values $<0,10$ were included in the multivariate analysis. Results: $12,4 \%$ had depression, $23,1 \%$ anxiety and $5,3 \%$ suicidality \%. In the multivariate model, an association was found between being divorced / widowed / separated $(P R=2, I 9 ; C l: I, I 8-4,07)$, being graduate $(P R=0,44 ; C l: 0,28-0,69)$, being undergraduate $(P R=0,26 ; C l: 0, I 4-0,48)$ and being unemployed $(P R=3,5 I ; C l: 0,79-$ $10,22)$ with depression. There was a significant association between female gender $\left(P R_{a}=1,62 ; C l: I, 25-2,09\right)$, being young $\left(P R_{a}=3,24 ; C l: I, 34-7,83\right)$, being an

Bachiller en Medicina Humana. Universidad Ricardo Palma, Lima, Perú. (ORCID: 0000-000I-8630-7502)

2 Médica pediatra. Instituto de Investigación en Ciencias Biomédicas, Universidad Ricardo Palma, Lima, Perú. (ORCID: 0000-000I-92052745)

3 Médico oncólogo clínico. Instituto de Investigación en Ciencias Biomédicas, Universidad Ricardo Palma, Lima, Perú. (ORCID: 00000002-5592-0504) 
adult $(P R=3,48 ; C l: I, 43-8,46)$, being graduate $(P R=0,44 ; C l: 0,33-0,58)$ and being independent employee $(P R=2,94 ; C l: I, 37-6,29)$ with anxiety. Female gender $\left(P R_{a}=0,48 ; C l: 0,27-0,85\right)$, being divorced / widowed / separated $\left(P R_{a}=4,69 ; C l: 2,23-9,83\right)$ and being dependent employee $(P R=0,24 ; C l: 0,10-0,57)$ were factors associated with suicidality. Conclusions: Occupation type was significantly associated with depression, anxiety and suicidality; gender with anxiety and suicidality; marital status with depression and suicidality; education level with depression and anxiety; and age range with anxiety.

Keywords: Depression, anxiety, suicidal ideation, social isolation, SARS-CoV-2 virus.

\section{INTRODUCCIÓN}

La cuarentena es la separación y restricción de movimiento de las personas que han estado potencialmente expuestas a una enfermedad contagiosa, como lo es la enfermedad del virus SARS-Cov2, con la finalidad de identificarla y atenuar el riesgo de infección. ${ }^{1}$ Esta definición es completamente diferente a la de "aislamiento social", que es la separación entre personas que han sido diagnosticadas con una enfermedad contagiosa y personas que no están enfermas; sin embargo, los dos términos se utilizan indistintamente a menudo por la población general. ${ }^{2}$

La palabra cuarentena se empleó por primera vez en Venecia, en 1127, debido a la lepra y fue ampliamente utilizada en respuesta a la peste negra, en el siglo XIV, aunque no fue hasta 300 años después que se utilizó como término adecuado ante la presencia de enfermedades masivas y altamente contagiosas. ${ }^{3}$ En el año 2020, la palabra cuarentena se ha vuelto a emplear como medida de salud pública por la Organización Mundial de la Salud (OMS) desde el inicio del brote del nuevo coronavirus causante del síndrome respiratorio agudo severo o SARS-CoV-2 (del inglés Severe Acute Respiratory Syndrome coronavirus 2) en Wuhan, China. ${ }^{4,5}$

Los coronavirus pertenecen a la familia Coronaviridae, que infectan tanto a animales (quirópteros y/o roedores) como a seres humanos. En los seres humanos, el coronavirus, puede causar una enfermedad leve similar a un resfriado común, mientras que otros causan enfermedades graves como el SARS, con el que comparte un genoma similar en $80 \%$, y el Síndrome Respiratorio de Medio Oriente o MERS.,

A medida que la población en general se vio más expuesta y vulnerable, la emergencia sanitaria y la crisis socioeconómica provocaron procesos disfuncionales $\mathrm{y}$ cambios desadaptativos en el estilo de vida que han incrementado las afecciones psiquiátricas con diferente nivel de gravedad. ${ }^{8}$

Vivir en cuarentena es una experiencia desagradable; así, la separación de los seres queridos, la pérdida de libertad, la incertidumbre sobre el estado de la enfermedad y el aburrimiento pueden crear efectos displacenteros., ${ }^{9,10}$ La actual pandemia por el virus SARS-CoV2 continúa generando reacciones psicológicas que pueden variar desde ansiedad leve a histeria colectiva, depresión severa e incluso comportamiento suicida o suicidio. ${ }^{11}$

Por lo mencionado, el objetivo del presente estudio fue identificar la frecuencia y los factores sociodemográficos asociados a depresión, ansiedad y suicidalidad en una población de Lima Metropolitana debido a la pandemia por el virus SARS-CoV2.

\section{MATERIAL Y MÉTODOS}

\section{Diseño del estudio}

Estudio cuantitativo, observacional, analítico de corte transversal mediante un muestreo no probabilístico en "bola de nieve". Debido a las medidas gubernamentales restrictivas de distanciamiento social, este estudio se realizó por medio de dos encuestas virtuales y anónimas, elaboradas en la plataforma Google Form que fueron difundidas por el aplicativo de mensajería instantánea para teléfonos inteligentes WhatsApp, Messenger y la red social Facebook. La difusión de las encuestas empezó con estudiantes y docentes universitarios y grupos religiosos; posteriormente los grupos mencionados reenviaron las encuestas a sus contactos.

Población y muestra

La población estuvo constituida por ciudadanos mayores de 18 años, residentes en Lima Metropolitana y que acataron el aislamiento social impuesto debido a la enfermedad producida por el virus SARS-CoV2 desde marzo de 2020. La muestra estuvo conformada por 1003 participantes que contaron con acceso a redes sociales (WhatsApp $y$ Facebook).

Variables e instrumentos

Las variables dependientes fueron depresión, ansiedad y suicidalidad. Se consideraron como variables independientes aquellas que pudieran estar vinculadas con este desenlace. Se obtuvo información en diferentes categorías sobre el género, edad (rango etario), estado civil, grado de instrucción y tipo de ocupación. Se recolectaron los datos mediante dos encuestas: en primer lugar, la Escala de Psicopatología Depresiva-6 (EPD-6) que consta de siete ítems, construida como una versión resumida de la Escala de Psicopatología Depresiva de 20 ítems para ser utilizada en tamizaje. El contenido de sus ítems corresponde a distintos síntomas depresivos y son puntuados en una escala de Likert de cuatro categorías en las seis primeras preguntas ("casi nunca/en ningún momento $=0$ puntos", "algunas veces/a veces = 1 punto", "frecuentemente/rara $\mathrm{vez}=2$ puntos" y "casi todo el tiempo/casi todos los días/ casi siempre $=3$ puntos"; excepto en las preguntas $\mathrm{N}^{\circ} 2$ y $\mathrm{N}^{\circ} 3$ en donde "si generalmente $=0$ puntos" y "no en ningún momento $=3$ puntos). El ítem 7 de la EPD-6 explora la presencia de suicidalidad, donde su puntuación es nominal (respuesta "a" = no presencia de suicidalidad; respuestas "b", "c", "d" ó "e" = presencia de suicidalidad). La validez fue de 0,89 a través del coeficiente de Pearson. Además, su índice de confiabilidad fue de 0,77 a través del alfa de Cronbach, con una sensibilidad del $80,2 \%$ y especificidad del $73,7 \%$ (punto de corte sugerido $\geq 8$ puntos). ${ }^{12}$

La segunda encuesta empleada fue la Escala de Ansiedad 
de Lima-20 (EAL-20), donde utilizaron 20 ítems de la Escala de Ansiedad de Lima original de 130 ítems. Se presentó en un formato de respuestas dicotómicas ("si $=1$ punto" y "no = 0 puntos"; excepto en la pregunta $\mathrm{N}^{\circ} 18$ en donde "si $=0$ puntos" y "no $=1$ punto"). Se consideraron indicadores referidos a síntomas psíquicos y físicos, y otros relacionados a la agorafobia. Los puntajes obtenidos con esta escala cuentan con un área bajo la curva ROC de 0,76. Asimismo, mostró un coeficiente de confiabilidad de 0,89 a través del alfa de Cronbach, una sensibilidad de 69,4\% y una especificidad de $67,5 \%$ (punto de corte sugerido $\geq 10$ puntos). ${ }^{13}$ Ambas encuestas fueron validadas en el Perú por Lozano-Vargas y Vega-Dienstmaier, en el año 2018, quienes buscaron disminuir el tiempo de aplicación sin perder la eficacia ni la predicción de sus antecesoras. ${ }^{12,13}$

Procedimiento y análisis estadístico

Los datos de las encuestas se descargaron de la plataforma Google Form y se exportaron a un archivo de Microsoft Excel 2019. Se utilizó el Stata versión 16.1. Se calcularon frecuencias y porcentajes de las variables independientes. Para la evaluación de la asociación de la depresión, ansiedad y suicidalidad con las variables independientes se empleó la prueba de Chi cuadrado con valores " $p$ " menores al 0,05. Se establecieron asociaciones mediante el cálculo de las razones de prevalencia (RP) con sus respectivos IC95\%. Las asociaciones ajustadas se calcularon conforme al modelo de regresión de Poisson con varianza robusta para cada uno de los grupos de variables asociadas a los desenlaces de interés. Las variables con valores de $\mathrm{p}<0,10$ se incluyeron en el modelo multivariado.

Consideraciones éticas

Se contó con la autorización del Comité de Ética en Investigación de la Facultad de Medicina Humana de la Universidad Ricardo Palma. La información que se obtuvo no atentó contra la privacidad e integridad de los participantes del estudio y durante la investigación se mantuvo el carácter confidencial de la información obtenida a través de la recolección de respuestas anónimas provenientes de las encuestas enviadas a través de las redes sociales.

\section{RESULTADOS}

De los 1003 participantes, predominó el sexo femenino con $64 \%$, el rango etario jóvenes de 18 a 29 años (56,7\%), el estado civil soltero $(70,1 \%)$, el grado de instrucción superior completo $(72,6 \%)$ y ser trabajador dependiente (45\%). (Tabla 1).

De los 1003 participantes, $232(23,1 \%)$ presentaron ansiedad (72,8\% mujeres y $27,2 \%$ hombres). Respecto al rango etario y ansiedad, $130(56,1 \%)$ eran jóvenes de 18 a 29 años, $98(42,2 \%)$ adultos de 30 a 59 años y $4(1,7 \%)$ adultos mayores. Referente al estado civil y ansiedad, 158 $(68,1 \%)$ eran solteros, $60(25,9 \%)$ convivientes/casados y $14(6 \%)$ divorciados/separados/viudos. En relación al grado de instrucción y ansiedad, $140(60,3 \%)$ fueron de grado de instrucción superior completo, $65(28,1 \%)$ de grado de instrucción superior incompleto y los que solo
Tabla I. Frecuencia de los factores sociodemográficas $(n=1003)$.

\begin{tabular}{|lll}
\hline Variables & $\mathbf{n}$ & $\%$ \\
\hline Género & & \\
\hline Femenino & 642 & 64,0 \\
\hline Masculino & 361 & 36,0 \\
\hline Rango etario & & \\
\hline Joven (I8 - 29 años) & 569 & 56,7 \\
\hline Adulto (30 - 59 años) & 381 & 38,0 \\
\hline Adulto Mayor (de 60 años a más) & 53 & 5,3 \\
\hline Estado Civil & & \\
\hline Soltero(a) & 703 & 70,1 \\
\hline Divorciado(a)/separado(a)/viudo(a) & 54 & 5,4 \\
\hline Conviviente/casado(a) & 246 & 24,5 \\
\hline Grado de Instrucción & & \\
\hline Superior completa & 728 & 72,6 \\
\hline Superior incompleta & 213 & 21,2 \\
\hline Secundaria & 62 & 6,2 \\
\hline Tipo de Ocupación & 258 & 25,7 \\
\hline Desocupado & 46 & 4,6 \\
\hline Trabajador independiente & & \\
\hline Trabajador dependiente & 24,7 \\
\hline Ama de casa & & \\
\hline
\end{tabular}

tenían secundaria eran 27 (11,6\%); por último, en relación al tipo de trabajo y ansiedad, $84(32,6 \%)$ eran trabajadores independientes, $83(35,8 \%)$ trabajadores dependientes, 59 $(25,4 \%)$ no tenían un trabajo y $6(2,6 \%)$ eran amas de casa. (Figura 1)

Del total de participantes, $124(12,4 \%)$ presentaron depresión (65,3\% mujeres y 34,7\% hombres). Respecto al rango etario y depresión, $73(58,9 \%)$ eran jóvenes de 18 a 29 años, 48 (38,7\%) adultos de 30 a 59 años y $3(2,4 \%)$ adultos mayores. Referente al estado civil y depresión, 90 $(72,6 \%)$ eran solteros, $21(16,9 \%)$ convivientes/casados y $13(10,5 \%)$ divorciados/separados/viudos. En relación al grado de instrucción y depresión, 89 (71,8\%) fueron de grado de instrucción superior completo, $17(13,7 \%)$ de

Figura I. Frecuencias de depresión, ansiedad y suicidalidad

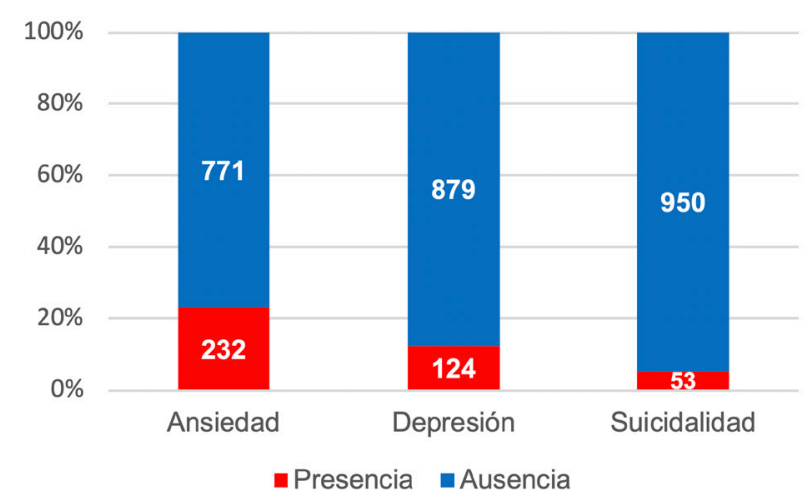


grado de instrucción superior incompleto y los que solo tenían secundaria eran 18 (14,5\%); por último, en relación al tipo de trabajo y depresión, $50(40,3 \%)$ no tenían un trabajo, $40(32,3 \%)$ trabajadores eran dependientes, 31 $(25 \%)$ trabajadores independientes, y $3(2,4 \%)$ eran amas de casa. (Figura 1)

De todos los participantes, $53(5,3 \%)$ presentaron suicidalidad (52,8\% mujeres y $47,2 \%$ hombres). Respecto al rango etario y suicidalidad, 28 (52,8\%) eran jóvenes de 18 a 29 años, $20(37,7 \%)$ adultos de 30 a 59 años y $5(9,5 \%)$ adultos mayores. Referente al estado civil y suicidalidad, $31(58,5 \%)$ eran solteros, $12(22,6 \%)$ convivientes/casados y $10(18,9 \%)$ divorciados/separados/viudos. En relación al grado de instrucción y suicidalidad, 39 (73,6\%) fueron de grado de instrucción superior completo, 11 (20,8\%) de grado de instrucción superior incompleto y los que solo tenían secundaria eran $3(5,6 \%)$; por último, en relación al tipo de trabajo y suicidalidad, $15(28,3 \%)$ eran trabajadores dependientes e independientes respectivamente, 18 (34\%) no tenían un trabajo y $5(9,4 \%)$ eran amas de casa. (Figura 1)

Con respecto a los análisis bivariados mostraron que ser divorciado(a) / separado(a) / viudo(a) se asoció con una probabilidad 1,82 veces mayor de presentar depresión, en comparación con ser conviviente / casado(a). En relación a los análisis bivariados del grado de instrucción, el tener un grado de instrucción superior completo e incompleto se asociaron con una probabilidad 0,58 y 0,31 veces menor de presentar depresión respectivamente, en comparación con solo haber cursado secundaria. El ser una persona sin trabajo también se asoció con una probabilidad 2,09 veces mayor de presentar depresión, en comparación con las amas de casa. Ser soltero solo se acerca a la asociación significativa $(\mathrm{p}<0,10)$ con una probabilidad 0,49 veces mayor de presentar depresión, en comparación con ser conviviente / casado(a). En el modelo multivariado, las variables que permanecieron asociadas significativamente $(p<0,05)$ con depresión fueron: ser divorciado(a) / separado(a) / viudo(a) $(\mathrm{RPa}=2,19$; IC: 1,18 - 4,07), los de grado de instrucción superior completo $(\mathrm{RPa}=0,44$; IC: $0,28-0,69)$ e incompleto $(\mathrm{RPa}=0,26$; IC: $0,14-0,48)$ y no tener trabajo $(\mathrm{RPa}=$ 3,51; IC: 0,79 - 10,22). Sin embargo, el ser soltero no llegó a asociarse significativamente $(\mathrm{p}=0,305)$ en el modelo multivariado ( $\mathrm{RPa}=1,27$; IC: 0,80 - 2,00). (Tabla 2)

En relación a ansiedad, los análisis bivariados mostraron que ser de género femenino se asoció con una probabilidad 0,5 veces mayor de presentar ansiedad, en comparación al género masculino. De igual manera el ser joven de 18 a

Tabla 2. Modelo de regresión de Poisson para factores sociodemográficos asociados a depresión.

\begin{tabular}{|c|c|c|c|c|c|c|c|c|}
\hline \multirow{2}{*}{$\begin{array}{l}\text { Factores } \\
\text { Sociodemográficos }\end{array}$} & \multirow{2}{*}{$\begin{array}{l}\begin{array}{l}\text { Con } \\
\text { depresión }\end{array} \\
n=124\end{array}$} & \multirow{2}{*}{$\begin{array}{l}\text { Sin } \\
\text { depresión } \\
n=879\end{array}$} & \multicolumn{3}{|c|}{ Modelo bivariado a } & \multicolumn{3}{|c|}{ Modelo multivariado ${ }^{b}$} \\
\hline & & & RPc & IC 95\% & Valor de $\mathrm{p}$ & $\mathrm{RPa}$ & IC $95 \%$ & Valor de $\mathrm{p}$ \\
\hline \multicolumn{9}{|l|}{ Género } \\
\hline Femenino & $81(12,6 \%)$ & $561(87,4 \%)$ & 1,05 & $0,74-1,49$ & 0,745 & -- & -- & -- \\
\hline Masculino & $43(11,9 \%)$ & $318(88,1 \%)$ & Ref & & & & & \\
\hline \multicolumn{9}{|l|}{ Rango etario } \\
\hline Joven (I8-29 años) & $73(12,8 \%)$ & $496(87,2 \%)$ & 2,26 & $0,73-6,94$ & 0,152 & -- & -- & -- \\
\hline Adulto (30-59 años) & $48(12,6 \%)$ & $333(87,4 \%)$ & 2,22 & $0,72-6,89$ & 0,166 & -- & -- & -- \\
\hline Adulto Mayor (de 60 años a más) & $3(5,7 \%)$ & $50(94,3 \%)$ & Ref & & & & & \\
\hline \multicolumn{9}{|l|}{ Estado civil } \\
\hline Soltero(a) & $90(12,8 \%)$ & $613(87,2 \%)$ & 1,49 & $0,95-2,35$ & 0,079 & 1,27 & $0,80-2,00$ & 0,305 \\
\hline Divorciado(a) / separado(a) / viudo(a) & $13(24,1 \%)$ & $4 \mathrm{I}(75,9 \%)$ & 2,82 & $1,50-5,27$ & 0,001 & 2,19 & $1,18-4,07$ & 0,013 \\
\hline Conviviente / casado(a) & $21(8,5 \%)$ & $225(91,5 \%)$ & Ref & & & Ref & & \\
\hline \multicolumn{9}{|l|}{ Grado de instrucción } \\
\hline Superior completo & $89(12,2 \%)$ & $639(87,8 \%)$ & 0,42 & $0,27-0,65$ & 0,000 & 0,44 & $0,28-0,69$ & 0,000 \\
\hline Superior incompleto & $17(8,0 \%)$ & $196(92,0 \%)$ & 0,27 & $0,15-0,50$ & 0,000 & 0,26 & $0,14-0,48$ & 0,000 \\
\hline Secundaria & $18(29,0 \%)$ & $44(71,0 \%)$ & Ref & & & Ref & & \\
\hline \multicolumn{9}{|l|}{ Tipo de ocupación } \\
\hline Desocupado & $50(20,2 \%)$ & 198 (79,8\%) & 3,09 & $1,00-9,49$ & 0,049 & 3,51 & $0,79-10,22$ & 0,035 \\
\hline Trabajador independiente & $31(12,0 \%)$ & $227(88,0 \%)$ & 1,84 & $0,59-5,78$ & 0,295 & 1,83 & $0,42-5,38$ & 0,309 \\
\hline Trabajador dependiente & $40(8,9 \%)$ & $4 I I(9 I, I \%)$ & 1,35 & $0,44-4,23$ & 0,595 & 1,45 & $0,31-4,15$ & 0,529 \\
\hline Ama de casa & $3(6,5 \%)$ & $43(93,5 \%)$ & Ref & & & Ref & & \\
\hline
\end{tabular}

a Regresión de Poisson simple con varianza robusta

${ }^{b}$ Regresión de Poisson múltiple con varianza robusta; modelo ajustado por estado civil, grado de instrucción y tipo de ocupación.

RPc: razón de prevalencia (crudo), RPa: razón de prevalencia (ajustado), IC 95\%: intervalo de confianza al $95 \%$. 
Tabla 3. Modelo de regresión de Poisson para factores sociodemográficos asociados a ansiedad.

\begin{tabular}{|c|c|c|c|c|c|c|c|c|}
\hline \multirow{2}{*}{$\begin{array}{l}\text { Factores } \\
\text { Sociodemográficos }\end{array}$} & \multirow{2}{*}{$\begin{array}{l}\text { Con } \\
\text { ansiedad } \\
n=232\end{array}$} & \multirow{2}{*}{$\begin{array}{l}\text { Sin } \\
\text { ansiedad } \\
n=77 \mid\end{array}$} & \multicolumn{3}{|c|}{ Modelo bivariado a } & \multicolumn{3}{|c|}{ Modelo multivariado b } \\
\hline & & & $\mathrm{RPc}$ & IC $95 \%$ & Valor de $\mathrm{p}$ & $\mathrm{RPa}$ & IC 95\% & Valor de $\mathrm{p}$ \\
\hline \multicolumn{9}{|l|}{ Género } \\
\hline Femenino & $169(26,3 \%)$ & $473(73,7 \%)$ & 1,50 & $1,16-1,95$ & 0,002 & 1,62 & $1,25-2,09$ & 0,000 \\
\hline Masculino & $63(17,5 \%)$ & $298(82,5 \%)$ & Ref & & & Ref & & \\
\hline \multicolumn{9}{|l|}{ Rango etario } \\
\hline Joven (I8-29 años) & I 30 (22,8\%) & $439(77,2 \%)$ & 3,02 & I,I6 - 7,86 & 0,023 & 3,24 & I,34 - 7,83 & 0,009 \\
\hline Adulto (30-59 años) & $98(25,7 \%)$ & $283(74,3 \%)$ & 3,40 & $1,30-8,88$ & 0,012 & 3,48 & $1,43-8,46$ & 0,006 \\
\hline Adulto Mayor (de 60 años a más) & $4(7,5 \%)$ & $49(92,5 \%)$ & Ref & & & Ref & & \\
\hline \multicolumn{9}{|l|}{ Estado civil } \\
\hline Soltero(a) & $158(22,5 \%)$ & $545(77,5 \%)$ & 0,92 & $0,71-1,19$ & 0,537 & -- & -- & -- \\
\hline Divorciado(a) / separado(a) / viudo(a) & 14 (25,9\%) & $40(74,1 \%)$ & 1,06 & $0,64-1,75$ & 0,812 & -- & -- & -- \\
\hline Conviviente / casado(a) & $60(24,4 \%)$ & $186(75,6 \%)$ & Ref & & & & & \\
\hline \multicolumn{9}{|l|}{ Grado de instrucción } \\
\hline Superior completo & 140 (I9,2\%) & $588(80,8 \%)$ & 0,44 & $0,32-0,60$ & 0,000 & 0,44 & $0,33-0,58$ & 0,000 \\
\hline Superior incompleto & $65(30,5 \%)$ & $148(69,5 \%)$ & 0,70 & $0,49-0,99$ & 0,046 & 0,75 & $0,54-1,05$ & 0,091 \\
\hline Secundaria & $27(43,6 \%)$ & $35(56,4 \%)$ & Ref & & & Ref & & \\
\hline \multicolumn{9}{|l|}{ Tipo de ocupación } \\
\hline Desocupado & $59(23,8 \%)$ & $189(76,2 \%)$ & 1,82 & $0,83-3,97$ & $0,|3|$ & 1,89 & $0,85-4,21$ & 0,120 \\
\hline Trabajador independiente & $84(32,6 \%)$ & $174(67,4 \%)$ & 2,49 & $\mathrm{I}, 15-5,37$ & 0,019 & 2,94 & $1,37-6,29$ & 0,005 \\
\hline Trabajador dependiente & $83(18,4 \%)$ & $368(8 \mathrm{I}, 6 \%)$ & $\mathrm{I}, 4 \mathrm{I}$ & $0,65-3,05$ & 0,382 & 1,63 & $0,74-3,60$ & 0,222 \\
\hline Ama de casa & $6(13,0 \%)$ & 40 (87,0\%) & Ref & & & Ref & & \\
\hline
\end{tabular}

29 años se asoció con una probabilidad 2,02 veces mayor de presentar ansiedad, en comparación con ser adulto mayor de 60 años a más. También ser adulto de 30 a 59 años se asoció con una probabilidad 2,4 veces mayor de presentar ansiedad, en comparación con los adultos mayores. Respecto al grado de instrucción, el tener un grado de instrucción superior completo e incompleto se asociaron con una probabilidad 0,56 y 0,3 veces menor de presentar ansiedad respectivamente, en comparación con solo haber cursado secundaria. El ser un trabajador independiente también se asoció con una probabilidad 1,49 veces mayor de presentar ansiedad, en comparación con las amas de casa. En el modelo multivariado, las variables que permanecieron asociadas significativamente $(p<0,05)$ con ansiedad fueron: ser de género femenino $(\mathrm{RPa}=1,62$; IC: 1,25 - 2,09), ser joven de 18 a 29 años $(\mathrm{RPa}=3,24$; IC: 1,34 - 7,83), ser adulto $(\mathrm{RPa}=3.48$; IC: $1,43-8,46)$, los de grado de instrucción superior completo $(\mathrm{RPa}=0,44$; IC: $0,33-0,58)$ y ser trabajador independiente $(\mathrm{RPa}=2,94$; IC: 1,37 - 6,29). Sin embargo, el tener un grado de instrucción superior incompleto no llegó a asociarse significativamente $(\mathrm{p}=0,091)$ en el modelo multivariado $(\mathrm{RPa}=0,75 ; \mathrm{IC}: 0,54$ - 1,05). (Tabla 3)
Con respecto a los análisis bivariados de suicidalidad, estos mostraron que ser divorciado(a) / separado(a) / viudo(a) se asoció con una probabilidad 2,8 veces mayor de presentar suicidalidad, en comparación con ser conviviente / casado(a). Del mismo modo el ser trabajador dependiente se asoció, con una probabilidad de 0,7 veces menor de presentar suicidalidad, en comparación con ser ama de casa. El género femenino solo se acerca a la asociación significativa $(p=0,084)$ con una probabilidad 0,4 veces menos de presentar suicidalidad, en comparación con ser de género masculino. En el modelo multivariado, las variables que permanecieron asociadas significativamente $(\mathrm{p}<0,05)$ con suicidalidad fueron: ser divorciado(a) / separado(a) / viudo(a) $(\mathrm{RPa}=4,69$; IC: 2,23 - 9,83) y ser trabajador dependiente $(\mathrm{RPa}=0,24$; IC: $0,10-0,57)$. Así mismo, el género femenino si llegó a asociarse significativamente $(\mathrm{p}=0,011)$ en el modelo multivariado $(\mathrm{RPa}=0,48 ; \mathrm{IC}: 0,27$ - 0,8). (Tabla 4).

\section{DISCUSIÓN}

Con la aplicación de nuevas medidas sanitarias a nivel nacional, especialmente la cuarentena y su repercusión en las actividades cotidianas, se esperaba que aumentasen los 
Tabla 4. Modelo de regresión de Poisson para factores sociodemográficos asociados a suicidalidad.

\begin{tabular}{|c|c|c|c|c|c|c|c|c|}
\hline \multirow{2}{*}{$\begin{array}{l}\text { Factores } \\
\text { Sociodemográficos }\end{array}$} & \multirow{2}{*}{$\begin{array}{l}\text { Con } \\
\text { suicidalidad } \\
n=53\end{array}$} & \multirow{2}{*}{$\begin{array}{l}\text { Sin } \\
\text { suicidalidad } \\
n=950\end{array}$} & \multicolumn{3}{|c|}{ Modelo bivariado a } & \multicolumn{3}{|c|}{ Modelo multivariado b } \\
\hline & & & $\mathrm{RPc}$ & IC (95\%) & $\begin{array}{l}\text { Valor } \\
\text { de } \mathrm{p}\end{array}$ & $\mathrm{RPa}$ & IC (95\%) & $\begin{array}{l}\text { Valor } \\
\text { de } \mathrm{p}\end{array}$ \\
\hline \multicolumn{9}{|l|}{ Género } \\
\hline Femenino & $28(4,4 \%)$ & $614(95,6 \%)$ & 0,63 & $0,37-1,06$ & 0,084 & 0,48 & $0,27-0,85$ & 0,011 \\
\hline Masculino & $25(6,9 \%)$ & $336(93,1 \%)$ & Ref & & & Ref & & \\
\hline \multicolumn{9}{|l|}{ Rango etario } \\
\hline Joven (I8-29 años) & $28(4,9 \%)$ & $54 \mid(95,1 \%)$ & 0,52 & $0,21-1,30$ & 0,161 & -- & -- & -- \\
\hline Adulto (30-59 años) & $20(5,3 \%)$ & 361 (94,7\%) & 0,56 & $0,22-1,42$ & 0,220 & -- & -- & -- \\
\hline Adulto Mayor (de 60 años a más) & $5(9,4 \%)$ & $48(90,6 \%)$ & Ref & & & & & \\
\hline \multicolumn{9}{|l|}{ Estado civil } \\
\hline Soltero(a) & $3 I(4,4 \%)$ & $672(95,6 \%)$ & 0,90 & $0,47-1,73$ & 0,761 & 0,92 & $0,43-2,00$ & 0,843 \\
\hline $\begin{array}{l}\text { Divorciado(a) / separado(a) / } \\
\text { viudo(a) }\end{array}$ & $10(18,5 \%)$ & $44(8 I, 5 \%)$ & 3,80 & $1,73-8,33$ & 0,001 & 4,69 & $2,23-9,83$ & 0,000 \\
\hline Conviviente / casado(a) & $12(4,9 \%)$ & $234(95,1 \%)$ & Ref & & & Ref & & \\
\hline \multicolumn{9}{|l|}{ Grado de instrucción } \\
\hline Superior completo & $39(5,4 \%)$ & $689(94,6 \%)$ & I, II & $0,35-3,48$ & 0,862 & -- & -- & -- \\
\hline Superior incompleto & II $(5,2 \%)$ & $202(94,8 \%)$ & $\mathrm{I}, 07$ & $0,31-3,71$ & 0,918 & -- & -- & -- \\
\hline Secundaria & $3(4,8 \%)$ & $59(95,2 \%)$ & Ref & & & & & \\
\hline \multicolumn{9}{|l|}{ Tipo de ocupación } \\
\hline Desocupado & $18(7,3 \%)$ & $230(92,7 \%)$ & 0,67 & $0,26-1,71$ & 0,400 & 0,74 & $0,29-1,86$ & 0,524 \\
\hline Trabajador independiente & $15(5,8 \%)$ & $243(94,2 \%)$ & 0,53 & $0,20-1,40$ & 0,203 & 0,45 & $0,19-1,11$ & 0,085 \\
\hline Trabajador dependiente & $15(3,3 \%)$ & $436(96,7 \%)$ & 0,31 & $0,12-0,80$ & 0,016 & 0,24 & $0,10-0,57$ & 0,001 \\
\hline Ama de casa & $5(10,9 \%)$ & $4 \mid(89,1 \%)$ & Ref & & & Ref & & \\
\hline
\end{tabular}

niveles de ansiedad, depresión y comportamiento suicida. La literatura internacional y la experiencia nacional respecto al manejo de la enfermedad por el virus SARSCoV2 permiten la toma de decisiones por parte de las instituciones gubernamentales en relación a cómo enfrentar la pandemia. Según el MINSA, al 14 de setiembre de 2021, el número de casos declarados positivos fue de 2162294 y el número de fallecidos es de 198840 personas, con una letalidad del $9,20 \%{ }^{14}$

El distanciamiento social, encierros y autoaislamiento a nivel nacional que fueron las medidas acatadas para reducir la propagación del virus SARS-CoV2 supuso que se incrementarían los problemas de salud mental en el país. Esta investigación corroboró lo mencionado ya que encontramos mayor porcentaje de ansiedad y depresión, con $23,1 \%$ y $12,4 \%$ respectivamente en la población estudiada, resultados similares a los reportados en el país al inicio de la pandemia en Cusco por Virto et al, 2021, con $20,7 \%$ y $18,1 \%$ de ansiedad y depresión respectivamente de un total de 1089 participantes. ${ }^{15}$ Sin embargo, en estudios internacionales en este mismo año reportaron lo contrario con predominio de la depresión respecto a la ansiedad, Alzueta et al, ${ }^{16}$ (25,4\% depresión y $19,5 \%$ ansiedad) y Wang et al $(17,17 \%$ depresión y $6,33 \%$ ansiedad $) .{ }^{17}$

Respecto al género, encontramos asociación de ansiedad con el género femenino $(\mathrm{p}=0,000)$ mas no con depresión $(p=0,745)$. En el año 2020 reportaron la misma asociación con este género, Özdin et $\mathrm{al}^{18}$ en Turquía $(\mathrm{OR}=2,47$; IC: 1,43 - 4,267) y Rossi et $\mathrm{al}^{19}$ en Italia, este último en una población de 18147 personas $(\mathrm{OR}=1,77$; IC: $1.59-1,97)$ y además reportaron asociación del género femenino con depresión ( $\mathrm{OR}=1,39$; IC: 1,24-1,56), lo cual no se encontró en nuestro estudio. (Ver Tabla 3)

Con respecto a la ansiedad, también se encontraron diferencias entre jóvenes y los otros grupos etarios. Estos resultados fueron similares a los obtenidos en estudios de Pakistán por Shah et $\mathrm{al}^{20}$, en 2021 y en Irlanda por Hyland et $\mathrm{al}^{21}$, en 2020; sin embargo, en este último estudio no se halló asociación significativa entre ser joven de 18 a 29 años con la ansiedad, contrario a nuestro hallazgo.

Al analizar las asociaciones considerando el grado de instrucción, en este estudio los participantes con grado de 
instrucción superior completo presentaron asociación con depresión y ansiedad en comparación con los participantes que solo cursaron secundaria. Estos resultados fueron opuestos a los reportados en el estudio iraní de Shahriarirad et $\mathrm{al}^{22}$, en 2021 , en donde el mayor nivel de educación no está asociado a depresión $(\mathrm{OR}=0,95$; IC: 0,87-1,04; $\mathrm{p}=0,313)$ ni a ansiedad (OR=1,07; IC: 0,96-1,19; $\mathrm{p}=0,182)$. En relación al tipo de trabajo, Verma et $\mathrm{al}^{23}$, en el año 2020 , evidenció en la población de India que el tener un empleo se asoció significativamente $(\mathrm{p}<0.05)$ con depresión $(\mathrm{OR}=1,91$; IC: $1,07-3,41)$ y ansiedad $(\mathrm{OR}=1,77$; IC: 1,00 $3,14)$; esto difiere parcialmente con nuestros resultados ya que encontramos que el estar sin empleo tuvo asociación significativa con depresión ( $\mathrm{p}=0,035)$, mas no con ansiedad $(\mathrm{p}=0,120)$.

Finalmente, este año en España, Mortier et $a^{24}$, respecto a la presencia de suicidalidad reportaron asociación significativa $(\mathrm{p}<0,005)$ con los factores de género femenino $(\mathrm{OR}=1,8 ; \mathrm{IC}$ 95\%: 1,3-2,4), estado civil divorciado/separado (OR=2,2; IC 95\%: $1,3-3,6)$ y estado civil viudo $(\mathrm{OR}=1,8$; IC 95\%: $1,0-3,3)$, resultados acordes a los nuestros.

La investigación contó con diversas limitaciones, como la muestra que se limitó a personas mayores de edad de la ciudad de Lima, por lo que se recomienda realizar nuevos trabajos para contrastarlos con nuestros resultados y, también, en otras regiones del país. Además, al ser este un estudio no probabilístico con un muestreo en "bola de nieve", los resultados estadísticos no son extrapolables a la población en general; sin embargo, fue un sondeo exploratorio del estado de la salud mental y que sienta las bases para investigaciones posteriores que generen aportaciones teóricas más amplias a partir de investigaciones con muestras de mayor tamaño y estudios probabilísticos o metaanálisis. Se recomienda continuar con estudios relacionados a este tema ya que aún nos encontramos en pandemia y con ciertas restricciones sociales.

El presente estudio concluye que la frecuencia de ansiedad, depresión y suicidalidad fue $23,1 \%, 12,4 \%$ y $5,3 \%$, respectivamente. Los factores asociados a depresión fueron: tipo de ocupación, grado de instrucción y estado civil; los asociados a ansiedad fueron: tipo de ocupación, grado de instrucción, género y rango etario; y, los factores asociados a suicidalidad fueron: tipo de ocupación, género y estado civil.

\section{REFERENCIAS BIBLIOGRÁFICAS}

I. Quarantine and Isolation | Quarantine | CDC [Internet]. [citado 16 de agosto de 202I]. Disponible en: https://www.cdc.gov/ quarantine/index.html

2. Manuell M-E, Cukor J. Mother Nature versus human nature: public compliance with evacuation and quarantine. Disasters. abril de 20I I;35(2):4I7-42. DOI: I0.IIII/j.I467-77I7.2010.0I219.x

3. Newman KLS. Shutt up: Bubonic plague and quarantine in early modern england. J Soc Hist. 2012;45(3):809-34. DOI: 10.1093/jsh/ shrl|4

4. 2019 Novel Coronavirus (2019-nCoV): Strategic Preparedness and Response Plan [Internet]. URL disponible en: https://www.who.int/ docs/defaultsource/coronaviruse/srp04022020.pdf?sfvrsn= $7 \mathrm{ff5} 5 \mathrm{ec} 0 \_4$

5. Prevención y control de infecciones durante la atención sanitaria de casos en los que se sospecha una infección por el nuevo coronavirus ( $\mathrm{nCoV}$ ). Orientaciones provisionales. Organización Mundial de la Salud 2020 [Internet]. URL disponible en: https://apps. who.int/iris/bitstream/handle/ I0665/330685/978924000 I I I4-spa. pdf

6. Wu F, Zhao S, Yu B, Chen Y-M, Wang W, Song Z-G, et al. A new coronavirus associated with human respiratory disease in China. Nature. 2020;579(7798):265-9. DOI: I0.1038/s4I 586-020-2008-3

7. Cui J, Li F, Shi Z-L. Origin and evolution of pathogenic coronaviruses. Nat Rev Microbiol. 2019;17(3):181-92. DOI:I0.1038/s4I579-0I8$0118-9$

8. Serafini G, Parmigiani B, Amerio A, Aguglia A, Sher L, Amore M. The psychological impact of COVID-19 on the mental health in the general population. QJM Int J Med. 2020; I I3(8):53 I-7. DOI: I0.1093/ qjmed/hcaa20I

9. Aspectos psicosociales de la pandemia COVID-19 en la población de la ciudad de Mendoza [Internet]. URL disponible en: https://www. redalyc.org/journal/3053/305366I59004/305366I59004.pdf

10. Barbisch D, Koenig KL, Shih F-Y. Is there a case for quarantine? Perspectives from SARS to Ebola. Disaster Med Public Health Prep. 20I5;9(5):547-53. DOI:10.10I7/dmp.20I5.38

II. Thakur V, Jain A. COVID 2019-suicides: A global psychological pandemic. Brain Behav Immun. 2020;88:952-3. DOI: 10.1016/j. bbi.2020.04.062

12. Vega-Dienstmaier JM. Construcción de versiones cortas de la Escala de Psicopatología Depresiva para tamizaje de depresión mayor y sus características psicométricas. Rev Neuro-Psiquiatr.20 I 8;8 I (3): I 54 64. DOI: I 0.20453/rnp.v8Ii3.3383

13. Lozano-Vargas A,Vega-Dienstmaier JM. Construcción y propiedades psicométricas de la Escala de Ansiedad de Lima de 20 ítems (EAL-20). Rev Neuro-Psiquiatr. 2018;8I(4):226-34. DOI:I0.20453/rnp. v8li4.3437

14. Covid 19 en el Perú - Ministerio del Salud [Internet]. [citado I5 de setiembre de 202I]. URL disponible en: https://covid I9.minsa. gob.pe/sala_situacional.asp

15. Virto Farfan C, Ccahuana F, Cornejo A, Loayza W,Alvarez B, Sanchez $D$, et al. Factores asociados al estrés, ansiedad y depresión en la etapa inicial del aislamiento social por COVID-19 (Sars-Cov-2), Cusco, Perú, 2020. 202I. DOI: I0.330 I7/ReCyTPeru202I.000I

16. Alzueta E, Perrin P, Baker FC, Caffarra S, Ramos-Usuga D, Yuksel D, et al. How the COVID-19 pandemic has changed our lives: A study of psychological correlates across 59 countries.J Clin Psychol. 202 I;77(3):556-70. DOI: I0.1002/jclp.23082

17. Wang Y, Di Y, Ye J, Wei W. Study on the public psychological states and its related factors during the outbreak of coronavirus disease 2019 (COVID-19) in some regions of China. Psychol Health Med. 202 I;26(I): I3-22. DOI: I0.1080/I3548506.2020.17468I7

18. Özdin S, Bayrak Özdin Ş. Levels and predictors of anxiety, depression and health anxiety during COVID-19 pandemic in Turkish society:The importance of gender. Int J Soc Psychiatry. 2020;66(5):504I I. DOI:I0.I I77/002076402092705 |

19. Rossi R, Socci V, Talevi D, Mensi S, Niolu C, Pacitti F, et al. COVID-19 Pandemic and lockdown measures impact on mental health among the general population in Italy. Front Psychiatry. 2020; I I:790. DOI: $10.3389 /$ fpsyt.2020.00790

20. Shah SMA, Mohammad D, Qureshi MFH, Abbas MZ, Aleem S. Prevalence, psychological responses and associated correlates of depression, anxiety and stress in a global population, during the coronavirus disease (COVID-19) pandemic. Community Ment Health J. 202।;57(I): I0I-I0. DOI: 10.1007/s I0597-020-00728-y

21. Hyland P, Shevlin M, McBride O, Murphy J, Karatzias T, Bentall RP, et al. Anxiety and depression in the Republic of Ireland during the COVID-19 pandemic. Acta Psychiatr Scand. 2020;142(3):249-56. DOI: 10.1 III/acps. I3219

22. Shahriarirad R, Erfani A, Ranjbar K, Bazrafshan A, Mirahmadizadeh A. The mental health impact of COVID-19 outbreak: a Nationwide Survey in Iran. Int J Ment Health Syst. 27202 I; I ( (I): I 9. DOI: I 0. I I86/ s| 3033-02 |-00445-3

23. Verma S, Mishra A. Depression, anxiety, and stress and sociodemographic correlates among general Indian public 
during COVID-19. Int J Soc Psychiatry. 2020;66(8):756-62. DOI: $10.1177 / 0020764020934508$

30. Mortier P, Vilagut G, Ferrer M, Alayo I, Bruffaerts R, CristóbalNarváez P, et al. Thirty-day suicidal thoughts and behaviours in the Spanish adult general population during the first wave of the Spain COVID-19 pandemic. Epidemiol Psychiatr Sci. 2021;30: el9. DOI: 10.1017/S2045796021000093

\section{CORRESPONDENCIA:}

Sergio Rolando Seminario Huallpa

srs.freiheit.9l@gmail.com
Fecha de recepción: 25-|0-202|.

Fecha de aceptación: 30-||-202|

Conflicto de interés: ninguno, según los autores.

Financiamiento: por los autores.

Contribuciones de los autores: SRSH y CLM han participado en la concepción y diseño del artículo, análisis e interpretación de datos y redacción del artículo; además SRSH ha participado en la recolección de datos. JCV ha participado en la revisión crítica del artículo y aprobación de la versión final, así como brindar aportes significativos en la discusión $y$ el manuscrito final. 